\title{
A Model-based Hybrid Approach for Circuit Breaker Prognostics Encompassing Dynamic Reliability and Uncertainty
}

\author{
Jose Ignacio Aizpurua, Victoria M. Catterson, Senior Member, IEEE, Ibrahim F. Abdulhadi, Maria Segovia Garcia
}

\begin{abstract}
Prognostics predictions estimate the remaining useful life of assets. This information enables the implementation of condition-based maintenance strategies by scheduling intervention when failure is imminent. Circuit breakers are key assets for the correct operation of the power network, fulfilling both a protection and a network reconfiguration role. Certain breakers will perform switching on a deterministic schedule, while operating stochastically in response to network faults. Both types of operation increase wear on the main contact, with high fault currents leading to more rapid ageing. This paper presents a hybrid approach for prognostics of circuit breakers, which integrates deterministic and stochastic operation through Piecewise Deterministic Markov Processes. The main contributions of this paper are (i) the integration of hybrid prognostics models with dynamic reliability concepts for a more accurate remaining useful life forecasting and (ii) the uncertain failure threshold modelling to integrate and propagate uncertain failure evaluation levels in the prognostics estimation process. Results show the effect of dynamic operation conditions on prognostics predictions and confirm the potential for its use within a condition-based maintenance strategy.
\end{abstract}

Index Terms-Prognostics, dynamic reliability, circuit breaker, uncertainty, hybrid model, physics of failure.

\begin{tabular}{ll} 
& \multicolumn{1}{c}{ Notation } \\
$S F_{6}$ & Sulphur hexafluoride \\
$I^{2} t$ & Cumulative degradation \\
$Z(t)$ & Piecewise Deterministic Markov Process \\
$\Psi(x, t)$ & Deterministic motion at position $x$ at time $t$ \\
$X_{n}$ & State of the system after the jump $n$ \\
$T_{n}$ & Time instant $n$ \\
$N\left(X_{n}, \cdot, \cdot\right)$ & Renewal Markov kernel \\
$s$ & Time instant in the process state space \\
$E$ & Probability space \\
$Q(z, \cdot)$ & Probability of transition from $z$ in $E \times E$ \\
$t_{p}$ & Prognostics prediction time instant \\
$\mathcal{R} \mathcal{U} \mathcal{L}_{t_{p}}$ & Remaining useful life at $t_{p}$ \\
$\mathcal{U}$ & Non-failed state of the system \\
$\overline{\mathcal{U}}$ & Failed state of the system \\
$\mathcal{L}(\theta \mid x)$ & Likelihood of a set of parameter values, $\theta$, \\
& given outcomes $x$
\end{tabular}

This work was supported by the EPSRC through grant number EP/M008320/1.

J. I. Aizpurua and V. M. Catterson are with the Institute of Energy and Environment, University of Strathclyde, Glasgow, UK (e-mail: jose.aizpurua@strath.ac.uk; v.m.catterson@strath.ac.uk).

I. F. Abdulhadi and M. Segovia Garcia are with the Power Networks Demonstration Center, University of Strathclyde, Glasgow, UK (e-mail: ibrahim.f.abdulhadi@strath.ac.uk; maria.segovia-garcia@strath.ac.uk).

$\begin{array}{ll}\mu_{y_{1}, \ldots, y_{n}} & \begin{array}{l}\text { Conditional distribution of } Z(t) \text { given available } \\ \text { observations }\left\{y_{1}, \ldots, y_{n}\right\}\end{array} \\ R_{z}(t) & \begin{array}{l}\text { Reliability of the system at time } t \text { when the } \\ \text { initial state is } z\end{array} \\ f(t) & \text { Probability Density Function (PDF) } \\ \Omega(t) & \text { Stress signal } \\ L(t) & \text { Ageing variable } \\ \beta & \text { Shape parameter of the Weibull distribution } \\ \eta & \text { Scale parameter of the Weibull distribution } \\ f_{\mathcal{U}} & \text { PDF of the failure threshold } \\ r_{\mathcal{U}} & \text { Randomly sampled failure threshold } \\ t_{j u m p} & \text { Jumping time or failure occurrence time } \\ t_{\text {trip }} & \text { Instant in which the Circuit Breaker (CB) } \\ & \text { receives open signal } \\ t_{\text {open }} & \text { Instant in which the CB reports being open } \\ t_{\text {switch }} & \text { CB switching operation start time } \\ t_{\text {end_switch }} & \text { CB switching operation finish time } \\ t_{\text {clear }} & \text { CB fault clearing operation open time } \\ i_{R M S} & \text { Current at the time of operation } \\ i_{\text {fault }} & \text { Fault current } \\ \mu_{\text {rep }} & \text { Constant repair rate } \\ D & \text { Total number of network fault shocks } \\ k_{1} & \text { Normalizing constant of the cumulative shocks } \\ k_{2} & \text { Normalizing constant of the delay } \\ s_{\text {shock }} & \text { Shock occurrence indicator variable }\end{array}$

\section{INTRODUCTION}

$\mathbf{T}$ HE INCREASING complexity of power networks including smart grid technologies requires efficient maintenance solutions to ensure the safe and cost-effective operation of its constituent assets [1]. Many of these assets are reaching the end of their life and condition-monitoring and life extension techniques are emerging as feasible technologies for enhanced maintenance planning of these systems (e.g., [2], [3]). The implementation of these approaches presents significant challenges for each specific asset.

Prognostics is the ability to acquire knowledge about events before they actually occur [4]. In engineering, failure prognostics aim to predict the Remaining Useful Life (RUL) of a component after a fault is diagnosed, i.e., the fault-to-failure progression of an asset (e.g., [2], [4], [5]).

Successful implementations of prognostic applications provide benefits for asset maintenance planning through the extension of the useful life and reduction of maintenance 
actions. Consequently, condition-based asset maintenance policies which integrate prognostics predictions when planning a maintenance schedule, can result in cost-effective operation of assets (e.g., [6], [7], [8]).

However, some assets prove particularly challenging when predicting failure, due to their operating regime incorporating both deterministic and stochastic behavior. This paper introduces a novel model-based hybrid approach to prognostics for such assets, with a particular focus on power network circuit breakers.

\section{A. Circuit Breaker Operation}

Circuit breakers are critical elements for the correct operation of the power network. Their objective is to interrupt current flow, and they operate either:

1) in response to a network fault - fault operation;

2) or to reconfigure the network (e.g., for switching in response to daily load changes) — switching operation.

Consider Fig. 1, where there are two paths for the generator to supply the load (along line 1, and along lines 2 and 3). At times of peak load, the current will flow along both paths. As the loading drops to a level where a single path has sufficient capacity for the current, circuit breakers A and B will operate to switch out one path. Traditionally, there is one peak in the load per day, so switching operations occur once per day.

However, if a fault occurs on line 1, circuit breakers A and $\mathrm{B}$ should operate to clear the fault and protect the rest of the network. A failure to operate will have significant safety and financial consequences, since the network fault will need to be cleared by a more distant circuit breaker, which takes longer and removes more customers from service. Accordingly, circuit breakers are required to have a very high availability.

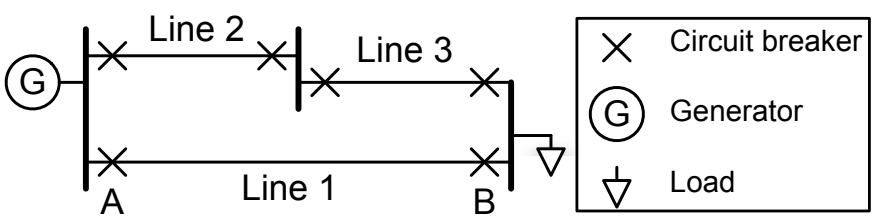

Fig. 1. A network requiring the operation of circuit breakers

Fig. 2 shows the cross section of a sulphur hexafluoride $\left(\mathrm{SF}_{6}\right)$ circuit breaker comprised of main and parallel arcing contact systems. The main contact conducts the current between the fixed and moving parts of the breaker and the arcing contact takes care of the arcing during separation. The nozzle guides $\mathrm{SF}_{6}$ gas to efficiently quench the arc.

The circuit breaker operates as follows [9], [10]:

- When the circuit breaker is in the closed position, the current flows from the fixed main contact to the moving main contact (Fig. 2, dashed-line empty arrowheads).

- When the circuit breaker begins to open as shown Fig. 2 , there is no arc as the current keeps flowing from the upper terminal to the moving parts through the fixed and moving arcing contacts (see current in Fig. 2, dashed-line filled arrowheads).

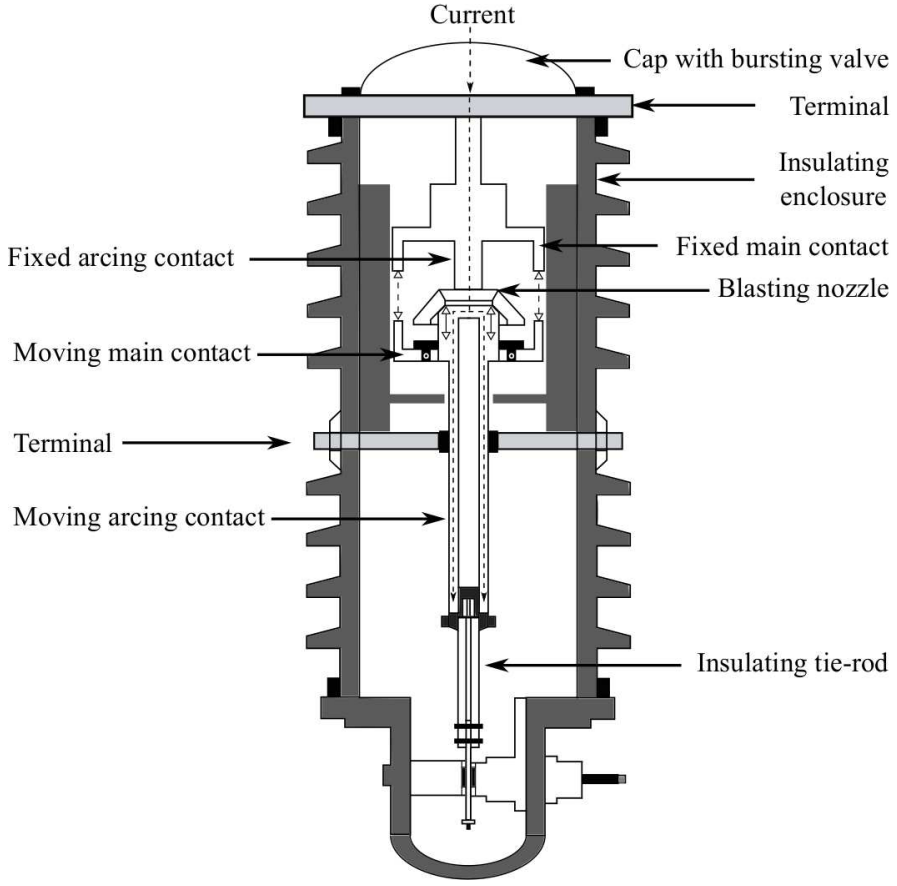

Fig. 2. Cross section of a circuit breaker derived from [9].

- During the opening of the moving arcing contacts (Fig. 2, solid-line empty arrowheads) the current keeps flowing due to the arc generated between the fixed and moving contacts.

- The arc is quenched when the circuit breaker is in the fully open position. In a high current situation (e.g., network fault) the puff of $\mathrm{SF}_{6}$ may be needed to quench the arc.

Each operation places wear on the main contact of the circuit breaker, which is proportional to the heating in the contact caused by the arc. This wear is referred to as $I^{2} t$, meaning the square of the current, $I$, multiplied by the time the arc is present, $t$ [11]. Every time the circuit breaker operates, more wear accumulates on the main contact. The total cumulative wear increases monotonically by the $I^{2} t$ of each operation.

Switching operations will tend to occur every day when the current is at the same relatively low level. Since a lower current can be broken more easily, the arc on the main contact will not exist for long, and $I, t$, and correspondingly $I^{2} t$ will be relatively low. Switching behavior is relatively deterministic, as it occurs under the same conditions each day.

However, whenever a network fault occurs, the current can increase by one or two orders of magnitude, and it takes longer to quench the subsequent arc. This situation places far higher $I^{2} t$ wear on the breaker. Different types of network fault, such as phase-to-phase faults, single phase-to-ground faults, and three phase-to-ground faults, will cause different levels of fault current to flow. Since the type and severity of a fault is stochastic, the value of $I^{2} t$ is also stochastic under fault conditions. 


\section{B. Circuit Breaker Failure Modes and Maintenance}

As described above, operation of a circuit breaker places cumulative wear on the main contact due to the presence of the arc. If not maintained, the circuit breaker would eventually reach a point of failure. Fail-to-open and fail-to-close are the main failure modes of circuit breakers and excessive contact wear is a critical event that directly causes the occurrence of these failure modes [12], [13]. For more detailed failure models of circuit breakers refer to [13], [14], [15].

In order to ensure current can be broken in a timely manner, breaker maintenance tends to be scheduled periodically based on the number of operations (see Subsection II-A). However, this preventive strategy does not take into account the dynamics of the system such as the different ageing effects of fault clearing operations versus switching operations. Therefore, it may result in a conservative (hence expensive) maintenance strategy which replaces assets even with a significant remaining useful life.

So as to implement intelligent maintenance policies, diagnostics and prediction steps are needed to reveal the actual state of the circuit breaker and predict its RUL, respectively [5]. Although the implementation of diagnostics techniques has been well studied for circuit breakers (see Subsection II-A for circuit breaker diagnostics examples), prognostics models for circuit breakers are scarce. Accordingly, so as to advance in the RUL prediction of circuit breakers, the main focus of this paper is on the prediction step.

In order to make prognostic predictions, it is necessary to create a model which replicates the future behavior of the circuit breaker and estimates the RUL from a specific time instant onwards. To this end, it is crucial to select a problem-specific prognostic prediction model depending on the system requirements, available engineering resources (runto-failure data or physics-of-failure engineering knowledge), and analyzed failure mode. See [16] for a prognostics modelselection process according to design requirements.

The main contact wear occurs due to both deterministic switching operation and stochastic network faults. Traditional mathematical processes such as Gamma or Wiener processes are not well suited for this problem because they model nonmonotonic and continuous monotonic degradation patterns respectively [16].

Model-based hybrid prognostic approaches have the capability to combine continuous and discrete time behavior of the system. This combination is suited to applications which need to consider a deterministic system behavior (e.g., switchingrelated degradation) where the deterioration is increased at discrete time instants (e.g., network faults).

\section{Contributions}

It is possible to modify existing hybrid prognostics prediction approaches (see Subsection II-B) in order to improve the remaining useful life prediction accuracy.

On the one hand, in order to model the future behavior of the asset, the discrete behaviour has been traditionally specified with an a priori determined stochastic model. It is possible to update this static model with deterministic operational signals (e.g., network loading) that modify the stochastic behavior according to the usage conditions. Dynamic reliability techniques are capable of handling component interactions and they constitute a more realistic system modelling for reliability analysis [17]. This way, it is possible to update the static failure model according to changes in the system operation, and accordingly replicate and predict the future behavior of the asset more accurately.

On the other hand, predicting the point in time at which the failure of an asset will occur requires the specification of a specific failure threshold. However, the definition of a deterministic failure threshold can be a difficult task for real applications. For a population of identical assets exposed to the same loading, some will fail sooner than others, and population failure time can be expected to conform to some distribution. In this paper, the specification of the failure threshold is included in the prognostics model as a Probability Density Function (PDF), and we propagate the effect of the uncertainty level to the final prognostic estimation.

Accordingly, the main contribution of this paper is the integration of a model-based hybrid prognostics model with dynamic reliability concepts for more accurate prognostics predictions of circuit breakers. The second contribution is the integration of an uncertain failure threshold level in the prognostics estimation.

\section{Organization}

Section II discusses this work in the context of other relevant work. Section III presents the generic proposed approach, while Section IV outlines its application to circuit breakers. Section V applies the proposed approach to a case study circuit breaker. Finally, Section VI draws conclusions and identifies future research challenges.

\section{RELEVANT WORK}

\section{A. Circuit Breaker Condition Monitoring}

An average size utility can have thousands of circuit breakers complicating their inspection and maintenance. Accordingly, electric utilities have traditionally performed preventive time-periodic maintenance programs to assess the condition of circuit breakers. The circuit breaker maintenance tends to be scheduled periodically based on number of operations [9], [10], [18]. Fig. 3 shows an example of operation-based maintenance program, where the remaining number of operations can be inferred from the current interrupted. For instance, if the current interrupted remains lower than $2500 \mathrm{~A}$ the estimated number of operations are 10000, however, it can decrease down to 100 operations when the current interrupted is 25 $\mathrm{kA}$.

The circuit breaker operation can be tracked through failure precursor variables indicating degradation, such as $\mathrm{SF}_{6}$ density, trip coil current profile or $I^{2} t$ cumulative degradation. These variables have been used to propose a number of circuit breaker data-mining and diagnostics approaches. The seminal work in [19] presented an automated circuit breaker diagnostics system based on control signals implemented through signal processing and expert system techniques. Similarly, a 


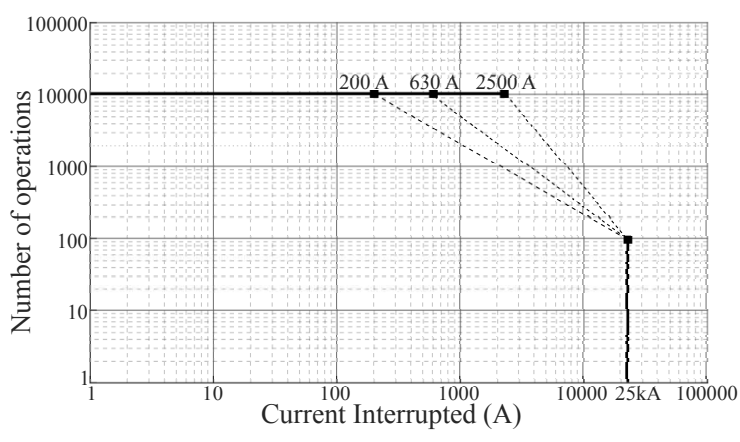

Fig. 3. Circuit breaker maintenance versus current interrupted [18].

data-mining process was proposed in [20] to derive data-driven diagnostic indicators from features of the trip coil current profile. This approach provides circuit breaker maintenance decision support combining classification techniques and expert interpretation.

Data-mining techniques for circuit breaker's condition analysis has also been applied more recently. Authors in [21] applied fuzzy set theory to analyse and integrate different circuit breaker operation and lifetime indices. Moreover evidential reasoning generates the overall assessment of the circuit breaker condition. A framework to assess the circuit breaker condition using the control signal waveforms was presented in [22]. The authors use classification techniques to categorize the health of the circuit breakers with qualitative (normal, alarm and emergency) and probabilistic quantitative indices.

The implementation of prognostics models is not as well studied as diagnostics techniques. As pointed out recently in [23], the ability to predict the ageing of circuit breakers is not fully developed, as they do not have a clearly defined physicsof-failure equation model. To the best of authors' knowledge, only previous results in [24] indicate that it is possible to establish a data-driven prognostics model to predict the RUL from $\mathrm{SF}_{6}$ density data samples.

In this paper we focus on developing a model-based prognostics approach to integrate engineering knowledge within the prediction model as in [25], instead of using data-driven prognostics prediction approaches (e.g., [6]). Accordingly, we analyze the deterioration of circuit breakers based on $I^{2} t$ cumulative degradation which requires considering simultaneously stochastic and deterministic operations.

\section{B. Hybrid Prognostics Approaches}

While a relatively new area, hybrid approaches have recently gained interest in the engineering prognostics arena (e.g., [26], [27]). Hybrid prognostics techniques integrate the continuous and discrete behavior of systems, reflecting a more realistic behavior of many industrial systems whose continuous-time dynamics are affected by periodic discrete events.

Piecewise Deterministic Markov Processes (PDMPs) provide a generic mathematical framework to model hybrid systems [28]. PDMPs represent deterministic trajectories interspersed with random jumps. The deterministic motion models the continuous phenomena (e.g., crack growth [26]) while the stochastic motion models shocks which influence the deterministic part of the system at random discrete times.

PDMPs have proven to be valid to create hybrid prognostics applications [26], [29]. These approaches assume that the system failure behavior is modelled with a shock model which is designed a priori. The shock models determine the future behavior of the system with discrete impacts on the deterministic motion and it is expressed with homogeneous Poisson processes with constant intensity. The approach in [29] adds the random evolution of the system to the stochastic shocks using continuous-time Markov chains.

PDMPs have also been used to solve dynamic reliability problems (e.g., [30], [31]). Instead of considering a priori established failure specifications of components, these approaches add the possibility to specify components with a variable failure rate which depends on the operating conditions of the system. This community has also started to create design tools to create hybrid models from user-friendly specifications, e.g., implementing PDMPs in Python [32] or linking reliability analysis and multi-physics specification tools [33]. There are other techniques which can also be used to solve dynamic reliability problems such as Dynamic Bayesian Networks [34], Stochastic Activity Networks [35], or Fluid Stochastic Petri Nets [36].

Apart from PDMP models, there are other hybrid prognostics approaches. A new formalism called Hybrid Particle Petri Nets combines Petri nets with particle filtering for health monitoring studies [37]. The model includes deterministic and stochastic properties and it is mainly focused on diagnostics.

Recently Daigle et al. proposed a generic model-based hybrid prognostics architecture inspired by hybrid bond graphs [27]. The continuous dynamics are defined with a model comprised of components, which in turn have variables with constraints. The discrete dynamics are defined with finite state machines that determine the switching behavior of each component. The prediction task focuses on simulating the model forward in time via Monte Carlo simulations until the event under study occurs.

In short, existing PDMP approaches for prognostics ([26], [29]) assume a static stochastic failure behavior and do not integrate the uncertainty that may surround the failure threshold specification. On the other hand, the reliability community has been using PDMPs to evaluate the system failure probability by dynamically updating the stochastic failure probability of systems with deterministic operational signals, but the system failure probability has not then been used to make prognostic predictions.

In this paper, we seek to improve prognostics predictions by combining dynamic reliability and uncertainty approaches with hybrid prognostics models. We focus on the use of PDMPs for failure prognostics of hybrid systems because it provides the flexibility to specify the ageing behavior of systems with any PDF, and dynamic reliability approaches can be integrated in a straightforward manner. 


\section{IntRoducing A NeW Hybrid PROGNOStiCS APPROACH}

This section introduces Piecewise Deterministic Markov Processes (PDMP), and shows how to combine prognostics, dynamic reliability techniques, and uncertainty in the failure threshold within a PDMP framework. Sections III-A and III-B review the existing literature on PDMPs and their use for prognostics, respectively. Section III-C introduces the extensions we propose for hybrid prognostics. After this, the following section applies this framework specifically to prognostics of circuit breaker ageing due to cumulative degradation.

\section{A. Preliminaries on PDMP}

A PDMP process $Z(t)$ couples a deterministic motion denoted with a function $\Psi$ and a jump process $\left(X_{n}, T_{n}\right)_{n \geq 0}$ where $X_{n}$ represents the state of the system after the jump $n$ at time $T_{n}$ [28].

As defined in [26], the jump process follows a Markov renewal process, where the next jump $\left(X_{n+1}, T_{n+1}-T_{n}\right)$ depends on the past only through the value of the last position $X_{n}$ and the process restarts at each renewal time $T_{i}$. This law is given by $N\left(X_{n}, \cdot, \cdot\right)$, where $N=(N(x, d z, d t))$ is called the renewal Markov kernel of the process [38].

Let us consider a probability space $(E, \varepsilon)$ which represents the values of the possible states of the system. As defined in [26], a process $Z$ with values in $E$ is a PDMP if it can be written as follows:

$$
Z(t)=\Psi\left(X_{n}, t-T_{n}\right), T_{n} \leq t<T_{n+1}
$$

with the following assumptions:

1) $\Psi(x, t+s)=\Psi(\Psi(x, t), s)$, for all $(s, t)$ and $s \rightarrow \Psi(x, s)$ is right continuous with left hand limits $\forall x$;

2) $\left(X_{n}, T_{n}\right)_{n \geq 0}$ is a Markov renewal process, with $T_{0}=$ 0 by convention, and with kernel $N(x, d z, d t)=$ $d F_{x}(t) Q(\Psi(x, t), d z)$ such as:

- $d F_{x}$ is the probability function of $\min \left(S_{x}, \alpha(x)\right)$ with

- $S_{x}$ random variable with hazard rate $b(\Psi(x, t))$,

- $\alpha(\mathrm{x}) \in \mathbb{R}_{+}$deterministic time such as $\alpha(\Psi(x, u))=\alpha(x)-u$

- Q is a probability of transition on $E \times E$.

The function $d F_{x}$ denotes the law of time before the next jump from position $x, Q(z, \cdot)$ represents the law of the position after a jump from position $z$, and $s$ denotes a time increment in the process state space. Full detail is given in [26].

\section{B. PDMP-based Prognostics}

Adhering to the formal notation in [26], the system degradation is modelled using a PDMP process denoted $Z=\left(Z_{t}\right)_{t \in \mathbb{R}_{+}}$ with values in $(\mathrm{E}, \varepsilon)$ where the random variable $Z_{t}$ represents the state of the system at time $t$.

Prognostics involves the prediction of the Remaining Useful Life (RUL). If $\mathcal{U}$ denotes the non-failed state of the system, then RUL at the prediction instant $\left(t_{p}\right), \mathcal{R} \mathcal{U} \mathcal{L}_{t_{p}}$, can be defined as the minimum time that the PDMP process $Z$ needs to reach the failed state in the process state space [26]. Formally,

$$
\mathcal{R} \mathcal{U} \mathcal{L}_{t_{p}}=\inf \left\{s \geq t_{p}, Z_{s} \notin \mathcal{U}\right\}-t_{p}
$$

The renewal process models damage events that occur repeatedly over time, and which further degrade the system until it reaches the failure state $\overline{\mathcal{U}}$. Renewals take place each time a damage event occurs and they accumulate damage on the system [38]. However, note that there is no asset repair until reaching the failure state $\overline{\mathcal{U}}$.

When using PDMP for prognostics, it is necessary to calculate the likelihood of the RUL prediction in (2) according to available observations. At discrete observation times $0<t_{1}<\ldots<t_{p}$ partial information of the system state is considered modelled by random variables $\left\{Y_{1}, \ldots, Y_{n}\right\}$. Assuming that observations $\left\{y_{1}, \ldots, y_{n}\right\}$ are available, the likelihood of the RUL prediction is defined as,

$$
\mathcal{L}\left(\mathcal{R} \mathcal{U} \mathcal{L}_{t_{p}} \mid Y_{1}=y_{1}, \ldots, Y_{n}=y_{n}\right)
$$

A possible solution of (3) involves a two-step methodology [26]. Firstly it is necessary to compute the conditional distribution of $Z_{t}$ for $t>t_{p}$ considering available observations $\left\{Y_{1}=y_{1}, \ldots, Y_{n}=y_{n}\right\}$ denoted as $\mu_{y_{1}, \ldots, y_{n}}(t)$ and defined as:

$$
\mu_{y_{1}, \ldots, y_{n}}(t)=\mathcal{L}\left(Z_{t} \mid Y_{1}=y_{1}, \ldots, Y_{n}=y_{n}\right)
$$

Secondly the reliability of the system is calculated when the initial state of the system is $z$, denoted $R_{z}(t)$, and defined as the probability of the process $Z$ being in a non-failed state:

$$
R_{z}(t)=\mathbb{P}(Z(s) \in \mathcal{U} \forall s \leq t)
$$

Finally, the RUL likelihood given observations up to the prediction time instant $t_{p}$ can be defined as follows:

$$
\mathbb{P}\left(\mathcal{R} \mathcal{U} \mathcal{L}_{t_{p}}>s \mid Y_{1}=y_{1}, \ldots, Y_{n}=y_{n}\right)=\int_{E} R_{z}(s) \mu_{y_{1}, \ldots y_{n}}\left(t_{p}\right) d z
$$

Therefore, the computation of the RUL distribution focuses on calculating the conditional distribution in (4) and quantifying the reliability in (5). When considering the different future conditions, only the second step is needed to recompute the RUL.

\section{Proposed Approach}

For PDMP-based hybrid prognostics models, the reliability model in (5) has been traditionally defined using a-priori defined shock model [26], [29]. Dynamic reliability techniques account for dynamic operation conditions and improve the accuracy of the reliability model (5) for time-varying operation conditions. Additionally, the specification of the failure state $\overline{\mathcal{U}}$ is surrounded by uncertainty criteria. This threshold determines the final RUL value (see (2)), but to the best of authors' knowledge this uncertainty has not been captured for hybrid prognostics modeling approaches.

Fig. 4 shows the proposed approach for the prognostics of hybrid systems, integrating the dynamic reliability and uncertainty criteria with the prognostics method from the literature. Next subsections explain each module in detail. 


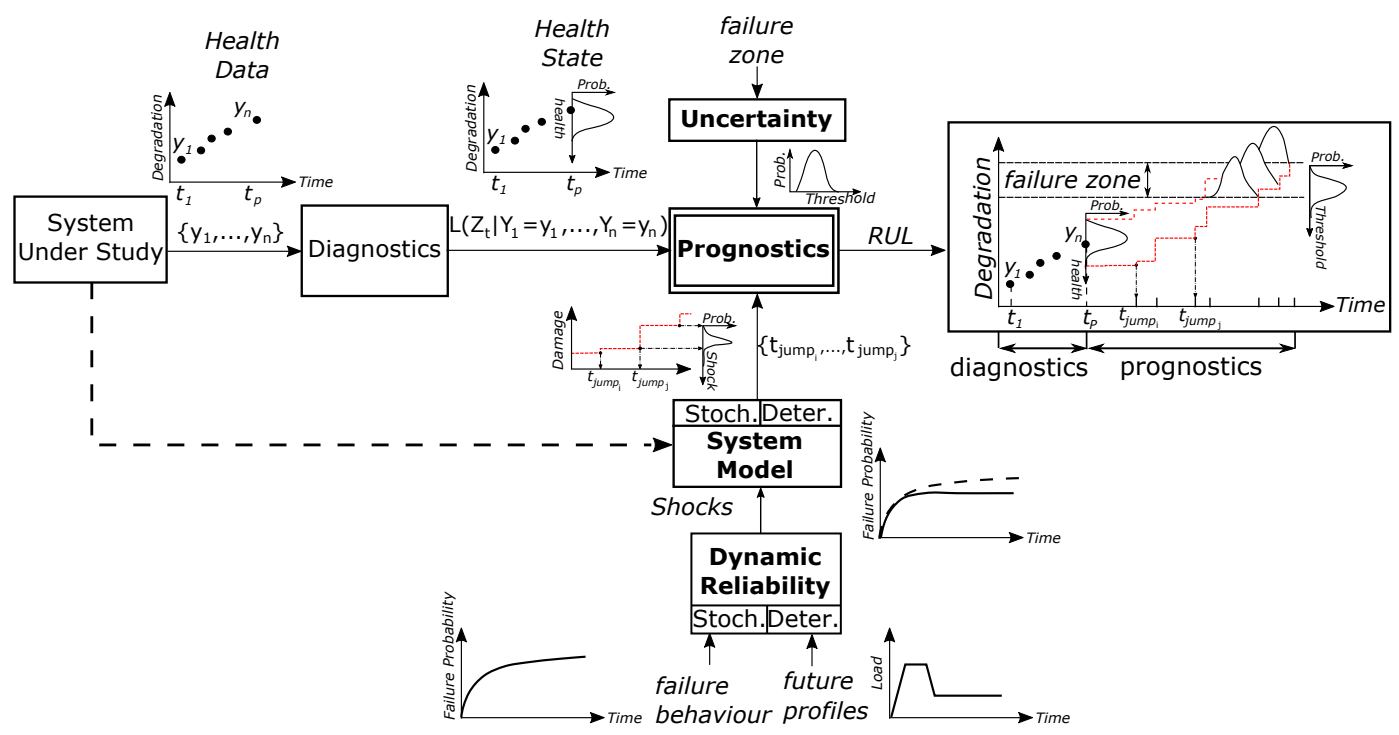

Fig. 4. Prognostics under uncertainty and dynamic reliability driven future evolution.

1) Prognostics: The prognostics module implements the model in (6) taking as input the likelihood of the actual system state (diagnosis), the uncertain failure threshold, and the future system behavior determined by the dynamic reliability and system model blocks.

The prognostics module not only predicts the distribution of the RUL of the asset under study (cf. Fig. 4), but it can also predict the evolution of a property of interest before reaching the failure threshold, e.g., remaining number of operations of a circuit breaker before reaching the failure threshold.

2) Diagnostics: As the system under study deteriorates it will go through several health states. The diagnostics module focuses on the current health state estimation given all the evidence up to the estimation time instant $t_{p}$.

For industrial systems, it is very difficult to physically assess the state of the system. Normally, this is diagnosed through the collected signals and knowledge of the system deterioration process. This process is not deterministic because there are noise terms involved in the data gathering process. Therefore the health state is represented with a probability density function. As indicated in (4): the system health state $\mu_{y_{1}, \ldots, y_{n}}(t)$ is estimated evaluating the likelihood of the collected signals $\left\{y_{1}, \ldots, y_{n}\right\}$ with respect to the system state $\left\{Y_{1}, \ldots, Y_{n}\right\}$ given the degradation process $Z_{t}$.

This module is commonly implemented using filtering techniques such as the Bayesian particle filter [2], [5], but it can be also implemented using other probabilistic state-estimation techniques such as Hidden Markov Models [39] or Dynamic Bayesian Networks [40].

3) System Model and Dynamic Reliability: The system model defines the degradation behaviour of the asset under study. This model includes deterministic and stochastic equations to define the behavior of the asset and inter-relationships between these equations, i.e., how shocks affect the deterministic motion and vice-versa (see Section IV for an example).

The dynamic reliability model predicts the random shock instants which impact the system model of the asset under study. Dynamic reliability approaches focus on updating dynamically the probability density function representing the system failure state according to operational conditions (e.g., [30]-[33]). For instance, the Weibull distribution allows the specification of a time-varying failure rate of the system $(\lambda(t))$ with the following density function:

$$
f(t)=\left(\frac{\beta}{\eta}\right) \cdot\left(\frac{t}{\eta}\right)^{\beta-1} \cdot e^{\left(\frac{-t}{\eta}\right)^{\beta}}
$$

where $\beta$ is the shape parameter and $\eta$ is the scale parameter.

It is possible to generalize this relationship with an ageing variable $L(t)$ that accounts for the real utilization of the asset under study [31]. In this paper, we extend this relationship for a general stress signal $\Omega(t)$ (e.g., the current a circuit breaker must interrupt) to be applied in prognostics studies (cf. (5)). The ageing process is defined with the differential equation:

$$
\frac{d L}{d t}=\Omega(t)
$$

Accordingly, we can redefine the variable failure rate and the PDF of the Weibull distribution changing the calendar time variable $t$ in (7) with the ageing variable $L(t)$ [31]:

$$
f(L(t))=\left(\frac{\beta}{\eta}\right) \cdot\left(\frac{L(t)}{\eta}\right)^{\beta-1} \cdot e^{\left(\frac{-L(t)}{\eta}\right)^{\beta}}
$$

where $L(t)$ is determined by the solution of (8).

The analytic solution of these systems is not trivial due to the non-linearities involved. A trade-off decision is needed between the complexity of the analytic solution and computational cost of simulations. In this paper we focus on simulation techniques based on the following basic reliability theory concepts [41].

The probability of a continuous random variable $X$ to take a value within $[a, b]$ can be defined with the integration of the Probability Density Function (PDF) $f_{x}(x)$,

$$
\mathbb{P}[a \leq X \leq b]=\int_{a}^{b} f_{x}(x) d x
$$


An alternative definition is the following:

$$
\mathbb{P}[t \leq X \leq t+\Delta t]=\int_{t}^{t+\Delta t} f_{x}(x) d x=f_{x}(t) \Delta t
$$

That is, if $\Delta t$ is an infinitely small period, the probability that $X$ is within $[t, t+\Delta t]$ is $f_{x}(t) \Delta t$.

In order to simulate the non-linear failure PDF which is dependent on non-linear equations, the approximation $f_{x}(t) \Delta t$ can be used to evaluate the failure probability and determine the failure occurrence time. Implementing stochastic Monte Carlo simulations, at each simulation step $\Delta t$ this condition is evaluated as in the inverse transform sampling method [31]:

$$
f_{x}(t) \Delta t \geq r
$$

where $r$ is a random number drawn from the uniform distribution $r \sim U([0,1])$.

The time instant at which the condition in (12) is satisfied represents the time at which the stochastic jump process occurs, and it is denoted $t_{j u m p}$. By the law of large numbers [42], $t_{\text {jump }}$ identifies PDF occurrence time and enables the approximation of the non-linear failure PDF such as in (9).

4) Failure Threshold Uncertainty: Prognostics applications are surrounded by uncertain information criteria that need to be integrated and propagated in the prediction model [43]. One specific example of interest is the definition of the failure zone. The exact specification of this threshold value is a complex task.

The PDF of the failure threshold $\left(f_{\mathcal{U}}(t)\right)$ indicates the uncertainty surrounding the failure threshold value. We have extended the calculation of RUL in (6) to include uncertainty criteria by adding a process to evaluate and classify predictions with randomly sampled failure threshold values as follows:

1) Random sampling: sample a failure threshold $r_{\mathcal{U}}$ randomly from the failure threshold PDF $f_{\mathcal{U}}(t)$.

2) RUL estimation: perform RUL computations with $r_{\mathcal{U}}$.

3) Classification: take the resultant PDF of the RUL estimation and classify the frequency of occurrence of the maximum RUL estimation.

The repeated execution of steps 1-3 generates a PDF (histogram) with the maximum failure probability RUL estimations. The classification of the results in step 3 can be done in different ways. For instance, it is possible to create a 3-dimensional plot which shows the different RUL PDF estimations for the different failure threshold values $r_{\mathcal{U}}$. However, as a more informative (and intuitive) representation, RUL prediction values with maximum probability have been used to create another distribution function (see Section V).

5) Benefits of the approach: The proposed approach provides a more accurate estimate of the remaining useful life of the asset under study (see next section for numerical examples). Potentially this estimation may have benefits for the maintenance of the asset under study through the implementation of fit-for-purpose maintenance planning strategies which can reduce maintenance costs by operating assets for longer with the assurance that it will not fail [44].
As shown in the next section with a circuit breaker case study, traditional approaches with periodical operation-based maintenance schedule [18] or approaches which use static and deterministic operation logic [26] estimate a conservative number of remaining number of operations. Interestingly, the proposed approach updates this estimate with real usage scenarios, and consequently it makes possible to adapt conservative calculations with more realistic figures improving the accuracy of the RUL estimation.

Depending on the real usage, the difference between dynamic reliability based prognostics predictions and traditional or static approaches varies. In the analysed case study, it is possible to run the asset under study longer with the assurance that it will not fail, and undertake maintenance actions less frequently. However, it may also be the case that the real usage is more stressed than traditional or static approaches, and this would lead to an earlier maintenance action.

In order to quantify the maintenance benefits a cost-benefit assessment is needed because the condition-based technologies and engineering efforts also incur costs. The cost assessment is beyond the scope of this paper. Please see [44] for the cost-benefit assessment of different maintenance strategies including the prognostics-updated predictive maintenance.

\section{Application to Circuit Breaker Prognostics}

The Cumulative Degradation (CD) of a circuit breaker is defined by the current in the arc between the fixed and moving contacts which forms while the breaker is opening (cf. Fig. 2). Formally it is defined as [11]:

$$
C D=\int_{t_{\text {trip }}}^{t_{o p e n}} i^{2} \cdot d t
$$

where $i$ is the instantaneous current and $t$ is the arc time. Since arc time is difficult to measure, it can be approximated by the time between the circuit breaker receiving the signal to open $\left(t_{\text {trip }}\right)$ and the time the breaker reports being open $\left(t_{\text {open }}\right)$.

The current that flows in the circuit breaker during its opening is different depending on the reason for its operation. Namely, current during switching operation is generally much lower than fault current. However, the effect of all operations is measured with the same three variables: shock instant, shock strength, and shock duration.

Network switching is the periodic operation of the circuit breaker in order to reconfigure the network. Its instantaneous effect on the cumulative degradation is small because the current (shock strength) is small and can be cleared quickly (shock duration). However, in the long run, reconfiguration in response to a daily load cycle can lead to a considerable number of network switching operations that contribute to the ageing of the circuit breaker. Hence, switching can be said to affect the cumulative degradation with periodic shock instants and a constant shock strength:

$$
i(t)= \begin{cases}i_{R M S} & \text { if } t=\left[t_{\text {switch }}, t_{\text {switch }}+t_{\text {end_switch }}\right] \\ 0 & \text { otherwise. }\end{cases}
$$


where $i_{R M S}$ is the root mean square current at the time of the operation, and $t_{\text {switch }}$ and $t_{\text {end_switch }}$ are periodic on/off times that determine the shock duration.

Fault clearing is a stochastic event that occurs when the electrical network protected by the circuit breaker experiences a fault. After the fault occurs, it is assumed that the circuit is returned to service with a constant exponential repair rate with parameter $\mu_{\text {rep }}$. The shock instant depends on the time of the network fault, which is specified with a PDF which depends on a stress signal. The stress signal $\Omega(t)$ modifies an a priori specified PDF of circuit reliability according to the network load. In this case, the chance of the circuit faulting is assumed to increase with load, but this property does not hold universally. For a rural network with a non-load-related reliability, for example, the circuit reliability PDF should be specified appropriately.

In order to calculate the future shock instants, we solve Eqs. (7)-(11) for each simulation step $\Delta t$ taking into account possible future stress profiles (daily load profiles) and checking if the acceptance condition in (12) is satisfied. If the acceptance test is passed, then we use the current time instant as the occurrence of a network fault denoted as $t_{j u m p}$.

The definition of a deterministic function which defines the fault current is not feasible for generically capturing the wide range of types of fault which may occur. However, based on engineering knowledge, we can define a current interval $\left[i_{A}, i_{B}\right]$ for a given circuit and sample randomly the fault current $i_{\text {fault }}$ according to $i_{\text {fault }} \sim U\left(\left[i_{A}, i_{B}\right]\right)$. This will determine the shock intensity of the fault clearing operation.

The open time of the circuit breaker for fault clearing can be defined with a random variable $t_{\text {clear }}$ which obeys the exponential distribution $t_{\text {clear }} \sim \exp (\lambda)$. It may be expected that repeated fault operation places particular stress on the circuit breaker, with a consequential effect on opening times. This can be modeled by (15), which takes account of the cumulative effect of fault current stress.

$$
\text { shock }=\sum_{d=1}^{d=D} \Omega\left(t_{j u m p}\right) / k_{1}
$$

where $D$ is the total number of network fault shocks, $k_{1}$ is a normalizing constant, and $\Omega\left(t_{\text {jump }}\right)$ is the stress signal evaluated at the shock instant $t_{j u m p}$.

These random shocks have an effect on the circuit breaker operation by increasing the shock duration and accordingly affecting the cumulative degradation. Therefore, we define the shock duration as shock duration $=t_{\text {clear }}+$ delay, where delay includes the effect of cumulative shocks on the open time and is defined as follows:

$$
\text { delay }=\sum_{d=1}^{d=D} \text { shock } \cdot e^{\text {shock }} / k_{2}
$$

where shock variable is computed in (15), $k_{2}$ is a normalizing constant, and $D$ is defined as in (15). For each shock instant, an extra delay is added to the open time of the circuit breaker.

Depending on the process under study, it is possible to tune the normalizing constants from historical delay data so as to match the effect of cumulative shock processes or they can be identified from real values (e.g., spring constant in a valve [26]). A possible extension of the shock model could be to analyse the effect of the number of circuit breaker operations on the failure PDF in (9) - see Section VI.

The stochastic events in (15) and (16) affect the deterministic motion in (13) at discrete time instants by modifying the shock intensity and duration. These equations are embedded in Fig. 4 as follows:

- The system model block embeds (13)-(16).

- The dynamic reliability formulation is given by (7)-(9) and the computation of the circuit failure time $\left(t_{j u m p}\right)$ is calculated with the condition in (12). The repair behavior of the circuit is defined with an exponential distribution with a constant repair rate $\mu_{\text {rep }}$.

- Diagnostics and uncertainty are expressed with PDFs $\mu_{y_{1}, \ldots, y_{n}}(t)$ and $f_{\mathcal{U}}(t)$ respectively.

Fig. 5 outlines the flowchart of the circuit breaker prognostics algorithm making use of the proposed approach.

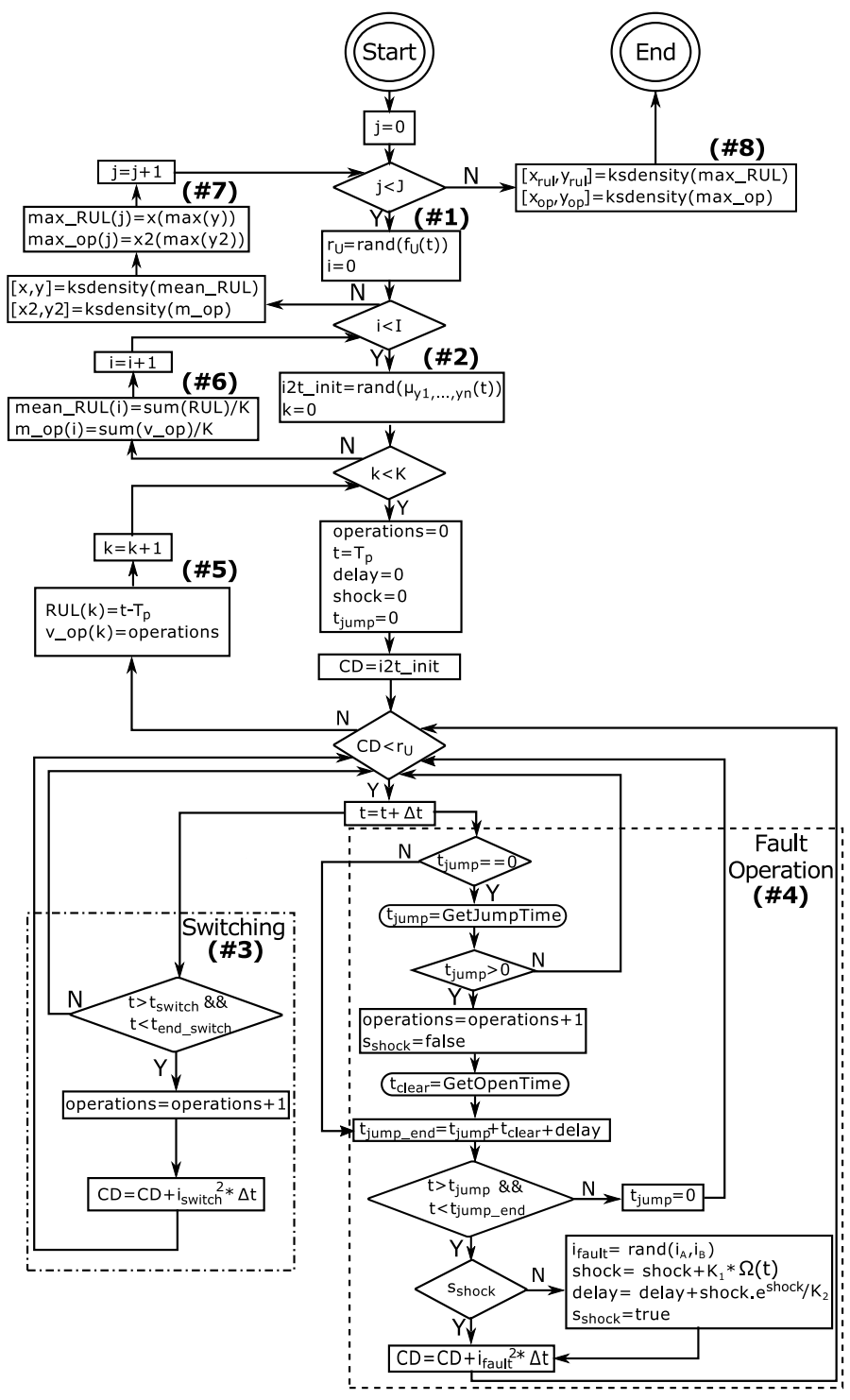

Fig. 5. Circuit breaker prognostics algorithm. 
Apart from the RUL estimation, we also calculate the remaining number of circuit breaker operations. To this end, we only change the time-dependent variables in (2) with number of operations and accordingly estimate the number of operations until reaching the failure threshold. The proposed approach involves the following steps:

\#1 Randomly sample the failure threshold $\left(r_{\mathcal{U}}\right)$ according to the PDF of the failure zone $f_{\mathcal{U}}(t)$.

\#2 Randomly sample the initial health state (i2t_init) according to the PDF of the actual health state $\mu_{y_{1}, \ldots, y_{n}}(t)$.

\#3 Check the network switching condition and if satisfied, increase the cumulative degradation and circuit breaker operation counter.

\#4 Check if a network fault has occurred:

- Obtain the jump time $t_{j u m p}$ with function GetJumpTime and check if $t_{\text {jump }}>0$. GetJumpTime implements the condition in (12), i.e., $t_{\text {jump }}>0$ iff the condition in (12) is true. If true:

- Calculate the stochastic open time for fault clearing $\left(t_{\text {clear }}\right)$ with function GetOpenTime using inversion sampling [42]: $t_{\text {clear }}=F^{-1}(r)$, where $F^{-1}$ is the quantile function and $r \sim U([0,1])$.

- For each shock occurrence (identified by the $s_{\text {shock }}$ variable) calculate its effect on the delay, increase the cumulative degradation accordingly, and increase the circuit breaker operation counter.

\#5 RUL calculation for the k-th Monte Carlo trial - cf. (2).

\#6 RUL and remaining circuit breaker operations probability calculation after $K$ Monte Carlo trials.

\#7 Classification of uncertainty results first creating a PDF with kernel density estimation [45] and then taking the RUL and remaining circuit breaker operation values with the maximum probability.

\#8 Estimate the PDF of the remaining useful life and remaining number of circuit breaker operations with randomly sampled failure threshold values.

\section{CASE Study}

The Power Networks Demonstration Centre (PNDC) is an $11 \mathrm{kV} / 400 \mathrm{~V}$ test facility for demonstrating smart grid technologies, located near Glasgow, UK. It was designed to accommodate significant levels of automation and communications, embedded generation, and fault-throwing capability, with the intention of trialling equipment and procedures for a distribution network of the future [46]. One of the $11 \mathrm{kV}$ circuit breakers at PNDC was selected for testing the hybrid prognostics approach. After conducting a workshop with engineers, Table I displays the variables for circuit breaker prognostics experiments and their values.

According to the fault levels experienced at PNDC (Table I, $i_{\text {fault }}$ ) and manufacturer specifications (Fig. 3) the standard maintenance strategy for the chosen breaker is to replace it every 10 years or after performing 10000 operations.

In an online context, the actual health of the circuit breaker can be monitored through $I^{2} t$ data samples $\left\{y_{1}, \ldots, y_{p}\right\}$, and predictions of RUL made on an on-going basis. For the
TABLE I

VARIABLES FOR CIRCUIT BREAKER PROGNOSTICS EXPERIMENTS

\begin{tabular}{|c|c|c|c|}
\hline Variable & Type & Distribution & Value \\
\hline$i_{\text {fault }}$ & stochastic & uniform & {$[35,250] \mathrm{A}$} \\
\hline circuit fault & stochastic & weibull & $\beta=36, \eta=100$ \\
\hline repair rate & stochastic & exponential & $\mu_{\text {rep }}=0.25$ \\
\hline$f_{\mathcal{U}}(t)$ & stochastic & normal & $\begin{array}{c}\text { mean=666667 hrs; } \\
\text { std. dev. }=400 \mathrm{hrs}\end{array}$ \\
\hline$t_{\text {clear }}$ & stochastic & exponential & $\lambda=1$ e5 \\
\hline $\begin{array}{c}\text { switching period } \\
\left(t_{\text {switch }}\right)\end{array}$ & constant & - & every $12 \mathrm{hrs}$ \\
\hline $\begin{array}{c}\text { switch. duration } \\
\left(t_{\text {end_switch }}\right)\end{array}$ & constant & - & $60 \mathrm{msec}$ \\
\hline$k_{1}$ & constant & - & 200 \\
\hline$k_{2}$ & constant & - & $1 e 9$ \\
\hline
\end{tabular}

purpose of this study, we assume a prediction time instant where the circuit breaker is diagnosed at $94 \%$ of the preplanned maintenance period. That is, the remaining number of circuit breaker operations is 600 or equivalently the remaining useful life is 0.6 years. The health state estimation indicated in (4) can be implemented using filtering techniques (see Subsection III-C2). Fig. 6 shows the estimated health state of the asset at the prediction time instant.

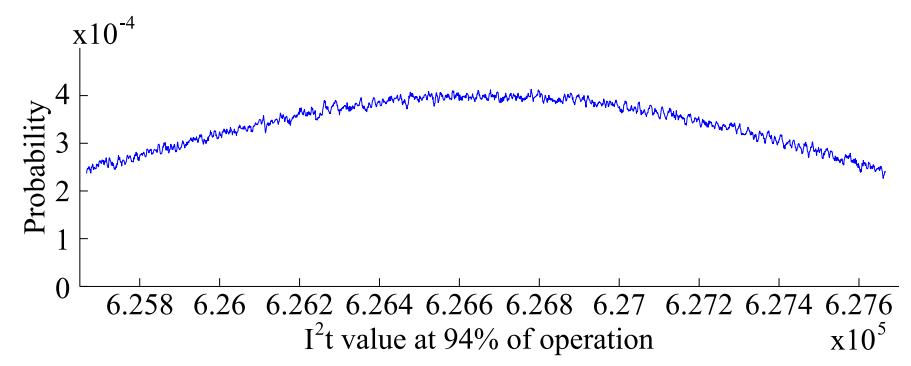

Fig. 6. $I^{2} t$ state at prediction time instant $t_{p}=9.4$ years

For future prediction instants, we compare the use of both static and dynamic reliability profiles. The static profile implements the shock instants according to a constant load profile, i.e., traditional PDMP-based prognostics prediction method (e.g., [26]). In contrast, dynamic reliability profiles modify the static behavior according to the applied load. Fig. 7 shows the different types of daily load profiles $(\Omega(t)$ in (8)).

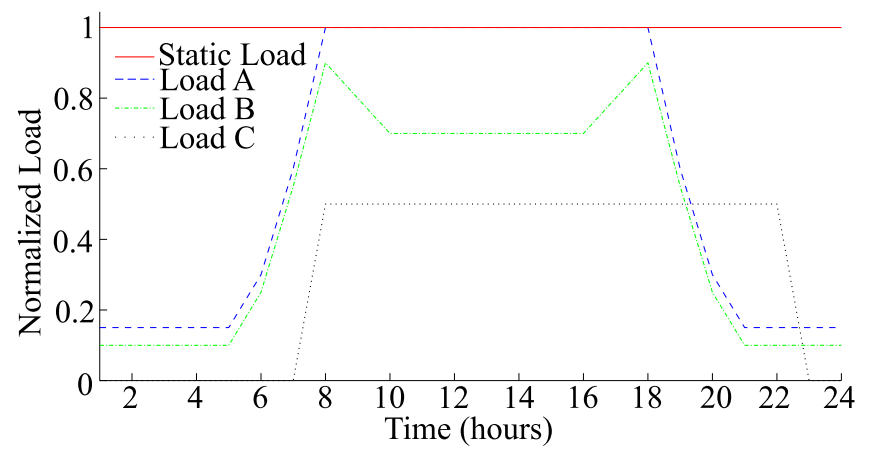

Fig. 7. Analyzed daily load profiles.

Different current load profiles impact the PDMP model in different ways and accordingly determine the future RUL and remaining number of circuit breaker operation values. 
Fig. 8 shows the effect of network fault events on the open time delay of the circuit breaker. Note that the delay increases only with network faults and not with switching operations.

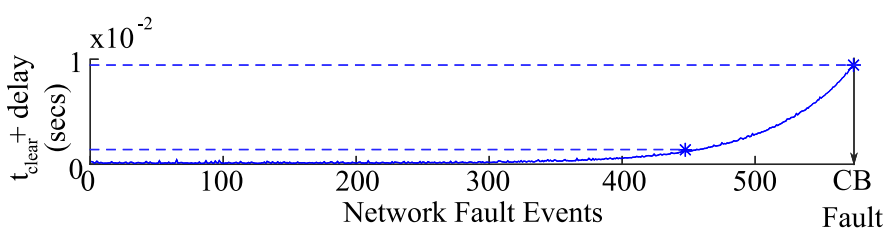

Fig. 8. Effect of network faults on the open time.

In Fig. 8 the circuit breaker switching duration starts to deviate from normal behavior after 450 further network fault events approximately (cf. low dashed line). After this threshold value, the circuit breaker starts to operate in a degraded mode owing to the cumulated network faults. These faults increase the delay exponentially (cf. (16)) until the circuit breaker reaches the failure limit of the cumulative degradation (cf. upper dashed line, $C B$ Fault event). Due to lack of real information, we have not taken into account previous cumulative delay. However, if the designer has this information, the accumulated delay should be added to the process in Fig. 8.

Taking a fixed failure threshold value (mean value of $f_{\mathcal{U}}(t)$ in Table I), Fig. 9 shows different predictions of the remaining number of operations according to the daily load profiles shown in Fig. 7. While the constant load profile specified according to the static reliability model shows the most conservative estimate, different predictions estimated with typical daily load profiles show an extended number of remaining circuit breaker operations. This highlights the importance of accurate load forecasting on circuit breaker prognostics.

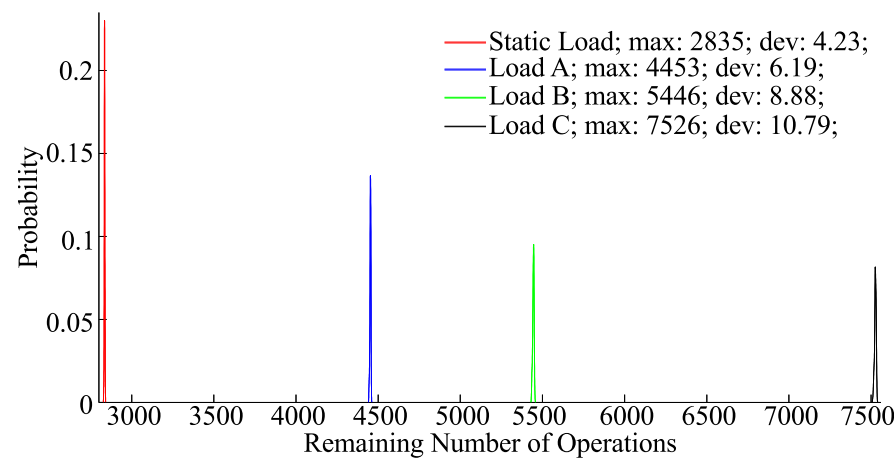

Fig. 9. Prediction of remaining number of operations.

Note that the static reliability driven prediction in Fig. 9 does not match with the pre-planned maintenance strategy of 600 remaining circuit breaker operations. This is because the default maintenance strategy implements an average estimation of cumulative degradation levels with fixed time intervals and constant current values. Using condition-based maintenance strategies, even with static reliability models, gives less pessimistic results. The static PDMP model includes random intervals for fault current and open time affected by random shocks. When we adapt the PDMP model with dynamic daily load profiles, we can see that the number of operations increases in proportion to the network load.
It should also be noted that the values in Fig. 9 show the remaining number of operations until circuit breaker failure, while maintenance strategies are required to adopt a safety margin for a timely maintenance [47].

The experiments in Fig. 9 have been performed with a fixed failure threshold. However, this assumption may not be realistic and we include uncertainty estimations based on the probability density function of the failure threshold, $f_{\mathcal{U}}(t)$. Taking the PDMP model with static load as a reference model and focusing on the remaining useful life prediction of the circuit breaker, Fig. 10 shows different estimations with randomly sampled failure threshold values (cf. Table I, $f_{\mathcal{U}}(t)$ ).

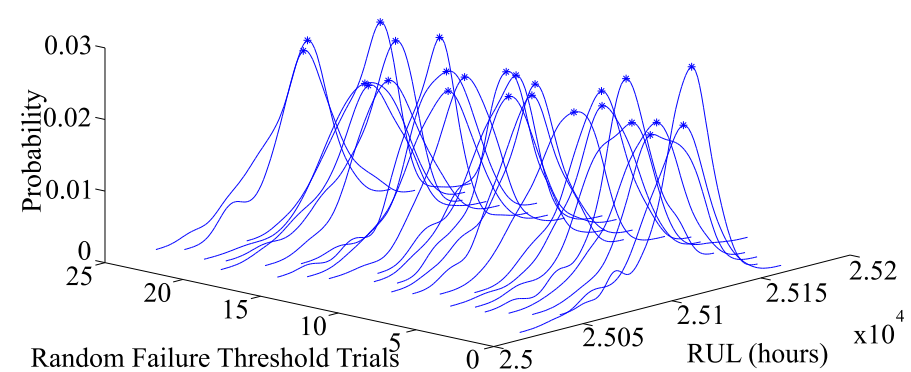

Fig. 10. RUL estimations with randomly sampled failure threshold values.

Each of the random failure threshold trials in Fig. 10 corresponds to a randomly sampled value from $f_{\mathcal{U}}(t)$. While this information is useful to see the effect of the failure threshold on the RUL prediction, as a more informative representation, we take the maximum likelihood value for each of the RUL estimations in Fig. 10 and then we apply kernel density estimation (cf. Subsection III-C and Fig. 5, step \#8). Fig. 11 shows the final PDF which integrates uncertainty criteria.

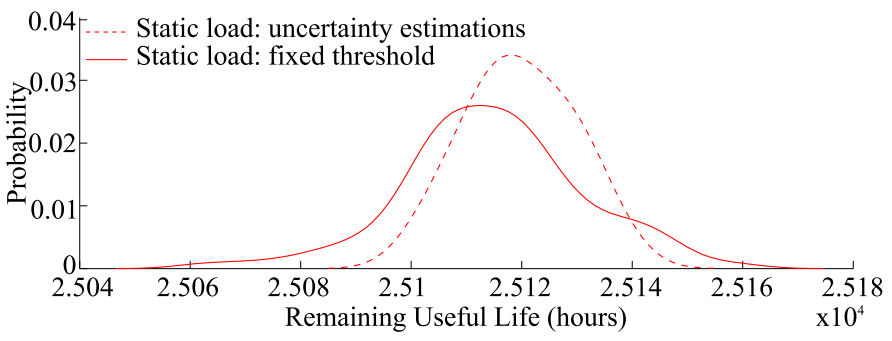

Fig. 11. RUL estimation with uncertainty prediction criteria.

Fig. 11 shows that when the uncertainty in the failure threshold is included, the shape and maximum likelihood values of the PDF of remaining useful life change accordingly. In this case, the prediction with failure threshold uncertainty information predicts an extended RUL value (dashed line) compared with the fixed failure threshold estimation (solid line).

The final effect of the uncertain failure threshold level on the RUL prediction depends on the adopted failure threshold distribution $f_{\mathcal{U}}(t)$. If the designer has a better informed knowledge of the failure threshold, fit-for-purpose failure threshold distributions may be used which can lead to more accurate results. 
As confirmed with the results shown in this section, the combined use of dynamic reliability and hybrid prognostics models improves the accuracy with respect to periodic operation-based maintenance schedules and hybrid prognostics models with static reliability models (Fig. 9). Besides, the integration of the uncertainty information in the failure threshold enables the evaluation of the effect of alternative failure threshold values on the final prediction estimations (Fig. 10) and prediction of the most likelihood useful life according to maximum RUL estimation values (Fig. 11).

While the models and algorithms presented in Section III are generic, Sections IV and V show the application of the proposed model to circuit breakers. The particular degradation equations and dynamic operation conditions depend on the asset under study. However, the theoretical framework can be generally applied to hybrid systems which degrade according to deterministic and stochastic events, with the particularity that the stochastic degradation process is modulated by deterministic signals, such as usage, load, or temperature.

In the case study we performed $10^{4}$ Monte Carlo iterations ( $K$ in Fig. 5), for each of the 100 random samples of the initial heath state ( $I$ in Fig. 5), and these were repeated for 25 randomly sampled failure threshold values ( $J$ in Fig. 5), which gives a total of $25 \times 10^{6}$ iterations.

The main limitation of the proposed approach is the simulation time. The lower the current load profile, the longer the simulation time. With a standard desktop Intel i7 with 8 cores, simulation times range from 8 hours (static load in Fig. 7) up to 240 hours (load $\mathrm{C}$ in Fig. 7). There are also other factors that affect the simulation time:

- Health state at prediction time: the better the health of the asset, the longer the simulation time.

- Failure threshold: the greater the failure threshold the longer the simulation time.

- Accuracy of the results: the higher the required accuracy, the higher the simulation time.

Although maintenance is normally planned months ahead of the failure occurrence, depending on the prognostics prediction horizon, the simulation time may be a critical factor. In order to alleviate long simulation periods, we have used Matlab's parallel computing toolbox. However, we also plan to study analytical techniques to speed-up the simulation time (see next section).

\section{CONCLUSIONS}

Circuit breakers are essential for the correct behavior of the power network. Circuit breakers exhibit a hybrid operation mode which integrates stochastic and deterministic operations. Accordingly we have presented a hybrid approach for prognostics of circuit breakers using Piecewise Deterministic Markov Processes.

The proposed approach extends existing hybrid prognostics techniques in two ways. On the one hand, it enables a more accurate remaining useful life forecast through integration with dynamic reliability techniques. That is, we update a priori defined fault conditions dynamically according to operation signals, e.g., daily load profiles of the power network. On the other hand, the proposed approach evaluates the effect of uncertain failure evaluation levels in the prognostics estimation process in order to handle the uncertainty that surrounds the specification of a failure threshold.

The presented approach enables fit-for-purpose maintenance planning for the circuit breaker, knowing that the predictions are tailored to the specific operational and failure circumstances of a given breaker. In the analysed case study, results show that the predictions incorporating dynamic reliability and uncertainty management lead to longer time horizon predictions of RUL, compared to predictions which do not use dynamic reliability concepts. However, the final prediction always depends on the specific usage of the breaker and knowledge of the failure threshold. This is why specific operational conditions and failure threshold specification have to be included on a case-by-case basis.

The confidence depends in part on the accuracy of forecasting the circuit load profile. While traditionally the load profile of a given circuit may remain relatively static over time, technologies associated with smart grids such as electric vehicles and demand-side management mean that loading is varying more than ever before. However, this presents another reason for tracking actual circuit breaker wear instead of planning maintenance on a periodic schedule.

Future work can address the following points:

- Validation of results: validate predictions with real runto-failure circuit breaker data.

- Shock models: analyse the effect of making the PDF of failure dependent on the number of operations.

- System-level prognostic prediction: integrate other failure modes (e.g., $\mathrm{SF}_{6}$ density, trip coil current) to obtain a complete prognostic model of the circuit breaker.

- Variable repair rates: apply dynamic reliability techniques to model condition-dependent repair rates, e.g., dependent on failed components or available repair resources.

- Simulation time: speed up simulations with dynamic stopping criteria such as in [48], where the number of Monte Carlo iterations are determined dynamically based on accuracy monitoring and confidence levels.

\section{ACKNOWLEDGMENT}

The authors would like to thank the editor and all the reviewers for their valuable comments that helped to improve the clarity and completeness of the paper.

\section{REFERENCES}

[1] F. Li, W. Qiao, H. Sun, H. Wan, J. Wang, Y. Xia, Z. Xu, and P. Zhang, "Smart transmission grid: Vision and framework," IEEE Transactions on Smart Grid, vol. 1, no. 2, pp. 168-177, Sept 2010.

[2] V. M. Catterson, J. Melone, and M. S. Garcia, "Prognostics of transformer paper insulation using statistical particle filtering of on-line data," IEEE Electrical Insulation Magazine, vol. 32, no. 1, pp. 28-33, Jan. 2016.

[3] A. D. Kenyon, V. M. Catterson, S. D. J. McArthur, and J. Twiddle, "An agent-based implementation of hidden markov models for gas turbine condition monitoring," IEEE Transactions on Systems, Man, and Cybernetics: Systems, vol. 44, no. 2, pp. 186-195, Feb 2014.

[4] G. Vachtsevanos, F. Lewis, M. Roemer, A. Hess, and B. Wu, Intelligent Fault Diagnosis and Prognosis for Engineering Systems. John Wiley \& Sons, Inc., 2006. 
[5] M. J. Daigle and K. Goebel, "Model-based prognostics with concurrent damage progression processes," IEEE Transactions on Systems, Man, and Cybernetics: Systems, vol. 43, no. 3, pp. 535-546, May 2013.

[6] S. J. Wu, N. Gebraeel, M. A. Lawley, and Y. Yih, "A neural network integrated decision support system for condition-based optimal predictive maintenance policy," IEEE Transactions on Systems, Man, and Cybernetics - Part A: Systems and Humans, vol. 37, no. 2, pp. 226-236, March 2007.

[7] G. Haddad, P. Sandborn, and M. Pecht, "An options approach for decision support of systems with prognostic capabilities," Reliability, IEEE Transactions on, vol. 61, no. 4, pp. 872-883, Dec 2012.

[8] K. T. Huynh, I. T. Castro, A. Barros, and C. Bérenguer, "On the use of mean residual life as a condition index for condition-based maintenance decision-making," IEEE Transactions on Systems, Man, and Cybernetics: Systems, vol. 44, no. 7, pp. 877-893, 2014.

[9] Megger, Circuit breaker testing guide. [Online]. Available: www.megger.com

[10] K. Harker, Power System Comissioning and Maintenance Practice. The Institution of Engineering and Technology, 1998.

[11] R. H. Kaufmann, "The magic of $\mathrm{I}^{2} \mathrm{t}$," IEEE Transactions on Industry and General Applications, vol. IGA-2, no. 5, pp. 384-392, Sept 1966.

[12] T. E. Wierman, D. M. Rasmuson, and N. B. Stockton, "Common-Cause Failure Event Insights - Circuit Breakers, NUREG/CR-6819,” Tech. Rep., 2003.

[13] G. D. Camps, "The development of a methodology to determina the maintenance strategy for high voltage circuit breakers," Ph.D. dissertation, University of Strathclyde, 2010.

[14] T. M. Lindquist, L. Bertling, and R. Eriksson, "Circuit breaker failure data and reliability modelling," IET Generation, Transmission Distribution, vol. 2, no. 6, pp. 813-820, November 2008.

[15] P. Choonhapran and G. Balzer, "Availability of hv circuit-breakers: The application of markov model," in Power Engineering Society General Meeting, 2007. IEEE, 2007, pp. 1-6.

[16] J. I. Aizpurua and V. Catterson, "Towards a methodology for design of prognostics systems," in Annual Conference of the Prognostics and Health Management Society, vol. 6, no. 22, 2015.

[17] P. Labeau, C. Smidts, and S. Swaminathan, "Dynamic reliability: towards an integrated platform for probabilistic risk assessment," Reliability Engineering \& System Safety, vol. 68, no. 3, pp. 219 - 254, 2000.

[18] Schneider Electric, GenieEvo range Metalclad, vacuum indoor switchgear. Installation, operation and maintenance instructions. [Online]. Available: http://goo.gl/pospcH

[19] M. Kezunovic, Z. Ren, G. Latisko, D. R. Sevcik, J. S. Lucey, W. E. Cook, and E. A. Koch, "Automated monitoring and analysis of circuit breaker operation," IEEE Transactions on Power Delivery, vol. 20, no. 3 , pp. 1910-1918, July 2005.

[20] S. Strachan, S. McArthur, B. Stephen, J. R. Mcdonald, and A. Campbell, "Providing decision support for the condition-based maintenance of circuit breakers through data mining of trip coil current signatures," Power Delivery, IEEE Trans. on, vol. 22, no. 1, pp. 178-186, Jan 2007.

[21] P. C. Lin, J. C. Gu, and M. T. Yang, "Intelligent maintenance model for condition assessment of circuit breakers using fuzzy set theory and evidential reasoning," IET Generation, Transmission Distribution, vol. 8 , no. 7, pp. 1244-1253, 2014.

[22] A. A. Razi-Kazemi, M. Vakilian, K. Niayesh, and M. Lehtonen, "Data mining of online diagnosed waveforms for probabilistic condition assessment of $\mathrm{SF}_{6}$ circuit breakers," IEEE Transactions on Power Delivery, vol. 30, no. 3, pp. 1354-1362, 2015.

[23] P. Westerlund, P. Hilber, T. Lindquist, and S. Kraftnat, "A review of methods for condition monitoring, surveys and statistical analyses of disconnectors and circuit breakers," in Int. Conf. on Probabilistic Methods Applied to Power Systems, July 2014, pp. 1-6.

[24] S. Rudd, V. Catterson, S. McArthur, and C. Johnstone, "Circuit breaker prognostics using $\mathrm{SF}_{6}$ data," in Power and Energy Society General Meeting, 2011 IEEE, July 2011, pp. 1-6.

[25] J. Luo, K. R. Pattipati, L. Qiao, and S. Chigusa, "Model-based prognostic techniques applied to a suspension system," IEEE Transactions on Systems, Man, and Cybernetics - Part A: Systems and Humans, vol. 38, no. 5, pp. 1156-1168, Sept 2008 .

[26] A. Lorton, M. Fouladirad, and A. Grall, "A methodology for probabilistic model-based prognosis," European Journal of Operational Research, vol. 225, no. 3, pp. $443-454,2013$.

[27] M. Daigle, I. Roychoudhury, and A. Bregon, "Model-based prognostics of hybrid systems," in Annual Conference of the Prognostics and Health Management Society, vol. 6, no. 12, 2015.

[28] M. H. A. Davis, Markov models and optimization, ser. Monographs on statistics and applied probability. Chapman \& Hall, 1993.
[29] D. N. Nguyen, L. Dieulle, and A. Grall, "Remaining useful lifetime prognosis of controlled systems: A case of stochastically deteriorating actuator," Mathematical Problems in Engineering, vol. 2015, 2015.

[30] J. Chiquet and N. Limnios, "A method to compute the transition function of a piecewise deterministic markov process with application to reliability," Stat. \& Prob. Lett., vol. 78, no. 12, pp. 1397 - 1403, 2008.

[31] F. Chiacchio, D. D'Urso, L. Compagno, M. Pennisi, F. Pappalardo, and G. Manno, "Shyfta, a stochastic hybrid fault tree automaton for the modelling and simulation of dynamic reliability problems," Expert Systems with Applications, vol. 47, pp. 42 - 57, 2016.

[32] H. Chraibi, "Dynamic reliability modeling and assessment with PyCATSHOO: application to a test case," in Probabilistic Safety Assessment and Management, PSAM, Tokyo, Japan, 2013.

[33] M. Bouissou and X. de Bossoreille, "From modelica models to dependability analysis," IFAC-PapersOnLine, vol. 48, no. 7, pp. 37 - 43, 2015.

[34] D. Codetta-Raiteri and L. Portinale, "Approaching dynamic reliability with predictive and diagnostic purposes by exploiting dynamic bayesian networks," Proceedings of the Institution of Mechanical Engineers, Part O: Journal of Risk and Reliability, 2014.

[35] D. Codetta-Raiteri, "Modelling and simulating a benchmark on dynamic reliability as a stochastic activity network," in European Modeling \& Simulation Symposium, EMSS, 2011, pp. 545-554.

[36] D. Codetta-Raiteri and A. Bobbio, "Solving dynamic reliability problems by means of ordinary and fluid stochastic petri nets," in European Safety and Reliability Conference, vol. 1, 2005, pp. 381-389.

[37] Q. Gaudel, E. Chanthery, and P. Ribot, "Hybrid particle petri nets for systems health monitoring under uncertainty," International Journal of Prognostics and Health Management, vol. 6, no. 22, p. 20, 2015.

[38] S. Ross, Introduction to Probability Models. Academic Press, 2006

[39] F. Camci and R. B. Chinnam, "Health-state estimation and prognostics in machining processes," IEEE Transactions on Automation Science and Engineering, vol. 7, no. 3, pp. 581-597, July 2010.

[40] D. C. Raiteri and L. Portinale, "Dynamic bayesian networks for fault detection, identification, and recovery in autonomous spacecraft," IEEE Trans. Systems, Man, and Cybernetics: Systems, vol. 45, no. 1, pp. 1324, 2015.

[41] M. Rausand and A. Høyland, System Reliability Theory: Models, Statistical Methods and Applications, Second Ed. Wiley-Interscience, 2003.

[42] E. Zio, The Monte Carlo Simulation Method for System Reliability and Risk Analysis. Springer Series in Reliability Engineering, 2013.

[43] S. Sankararaman, "Significance, interpretation, and quantification of uncertainty in prognostics and remaining useful life prediction," Mech. Syst. \& Signal Process., vol. 5253, pp. 228 - 247, 2015.

[44] J. I. Aizpurua, V. M. Catterson, F. Chiacchio, and D. D'Urso, “A costbenefit approach for the evaluation of prognostics-updated maintenance strategies in complex dynamic systems," in Proceedings of the European Safety \& Reliability Conference (ESREL'16), Glasgow, UK, 2016.

[45] J. S. Kim and C. Scott, "Robust kernel density estimation," in Acoustics, Speech and Signal Processing, 2008. ICASSP 2008. IEEE International Conference on, 2008, pp. 3381-3384.

[46] C. Booth, F. Coffele, and G. Burt, "The power networks demonstration centre: An environment for accelerated testing, demonstration and validation of existing and novel protection and automation systems," in IET Int. Conf. on Developments in Power System Protection, 2014, pp. 1-6.

[47] J. I. Aizpurua and V. M. Catterson, "On the use of probabilistic modelchecking for the verification of prognostics applications," in IEEE Int. Conf. on Intelligent Computing and Information Systems, Dec 2015, pp. 7-13.

[48] I. Meedeniya, A. Aleti, and L. Grunske, "Architecture-driven reliability optimization with uncertain model parameters," Journal of Systems and Software, vol. 85 , no. 10 , pp. $2340-2355,2012$

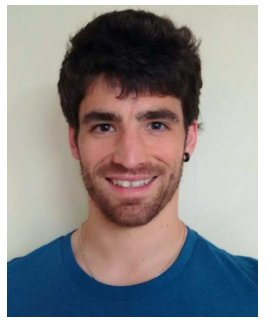

Jose Ignacio Aizpurua is a Research Associate within the Institute for Energy and Environment at the University of Strathclyde, Scotland, UK. He received his Eng., M.Sc., and Ph.D. degrees from Mondragon University (Spain) in 2010, 2012, and 2015 , respectively. He was a visiting researcher in the Dependable Systems Research Group at the University of Hull (UK) during autumn 2014. His research interests include prognostics, dependability, condition monitoring, and systems engineering. 


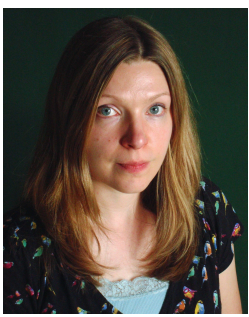

Victoria M. Catterson is a Senior Lecturer within the Institute for Energy and Environment at the University of Strathclyde, Scotland, UK. She received her B.Eng. (Hons) and Ph.D. degrees from the University of Strathclyde in 2003 and 2007 respectively. Her research interests include condition monitoring, diagnostics, and prognostics for power engineering applications.

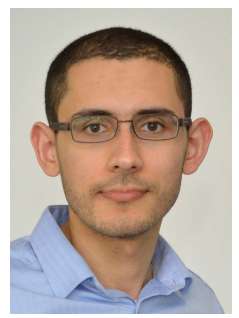

Ibrahim Abdulhadi received the M.Eng. and Ph.D. degrees in electronic and electrical engineering from the University of Strathclyde, UK, in 2007 and 2013. $\mathrm{He}$ is currently an R\&D Engineer within the universitys Power Networks Demonstration Centre. He has experience working for UK distribution and transmission network operators. His research interests include power system protection, real-time power system simulation, hardware in the loop testing and communications applications in smart grids.

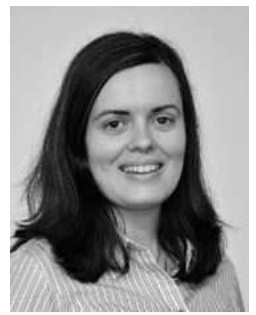

Maria Segovia-Garcia is a Knowledge Exchange Associate at the Power Networks Demonstration Centre (PNDC), University of Strathclyde. She received her Ph.D. in 2009 from the University of Granada (Spain). She worked as a postdoc at the Nuclear Metrology Department at the Free University of Brussels (ULB) before joining the PNDC in 2013. Her research interest include modelling assets deterioration, estimation of maintenance actions impact and data analysis. 